\title{
Closed extensor tendon rupture caused by Kienböck disease: a case report
}

\author{
Jong Yun Choi, Won Jin Cha, Ee Room Jung, Bommie F. Seo, Sung-No Jung \\ Department of Plastic and Reconstructive Surgery, College of Medicine, The Catholic University of Korea, Seoul, Korea
}

Kienböck disease, a rare disease that can cause chronic pain and motor dysfunction, occurs due to avascular necrosis of the lunate bone, which leads to dislocation of the carpal bone. Among various other etiologies, Kienböck disease can cause closed tendon rupture of the finger. In this report, we introduce a case of total rupture of the second extensor digitorum communis and the extensor indicis proprius tendons caused by undiagnosed Kienböck disease in an elderly female patient.

Keywords Tendon injuries / Osteonecrosis / Tendon transfer / Lunate bone / Caes reports

\author{
Correspondence: Sung-No Jung \\ Department of Plastic and \\ Reconstructive Surgery, Uijeongbu St. \\ Mary's Hospital, College of Medicine, \\ The Catholic University of Korea, 271 \\ Cheonbo-ro, Uijeongbu 11765, Korea \\ Tel: +82-31-820-3935 \\ Fax: +82-31-874-0301 \\ E-mail: jsn7190@catholic.ac.kr
}

Received: August 10, 2021 - Revised: October 12, 2021 - Accepted: October 22, 2021

pISSN: 2234-6163 • elSSN: 2234-6171 • https://doi.org/10.5999/aps.2021.01522 • Arch Plast Surg 2022;49:76-79

\section{INTRODUCTION}

Kienböck disease is rare, has a male preponderance of $3: 1$ to 7:1, and occurs mainly from adolescence to middle age. Closed tendon rupture of the hand can occur due to several causes including traumatic tendon avulsion, spontaneous rupture, attritional rupture, infiltrative tenosynovial rupture, and iatrogenic causes [1]. Kienböck disease results from avascular necrosis of the lunate bone and leads to dislocation of the carpal bone. It can cause closed tendon rupture by attritional injury of the tendon due to a collapsed fragment of the lunate bone [2]. Closed rupture of the extensor tendons secondary to necrosis and collapse of the lunate bone mainly affects the extensor indicis proprius (EIP) of the index finger and the fourth compartment of the extensor tendons [3]. Herein, we present a case of total rupture of the second extensor digitorum communis (EDC) and the EIP tendon caused by undiagnosed Kienböck disease in an elderly female farmer.

\section{CASE}

A 75-year-old right-handed woman, who had underlying diseases including diabetes, heart failure, hypertension, and asthma, visited the outpatient clinic because she was not able to extend her left index finger, a symptom that had abruptly started 2 weeks previously (Fig. 1). She also complained of a tingling sensation that had lasted for 5 years, and her wrist would occasionally swell, accompanied by severe pain every morning. As a farmer, she usually pulled up radishes from the field using both hands. She sought conservative physical therapy from a local clinic, but her symptoms did not improve. Plain radiographs demonstrated negative ulnar variance with a sclerotic collapsed lunate bone, suggestive of stage IV in Lichtman classification, and osteoarthritis in the left second distal interphalangeal joint. Magnetic resonance imaging (MRI) showed complete tearing of the EIP and second EDC tendons with retraction and dorsal displacement of the lunate fragment (Fig. 2). A nerve conduction study showed median nerve entrapment, which suggested carpal tunnel syndrome. 


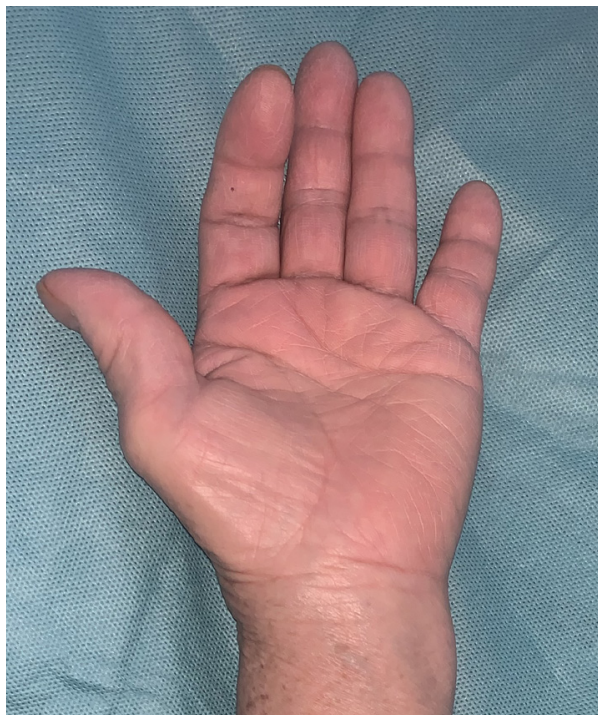

Fig. 1. Initial clinical photograph: the left hand of a patient with Kienböck disease.
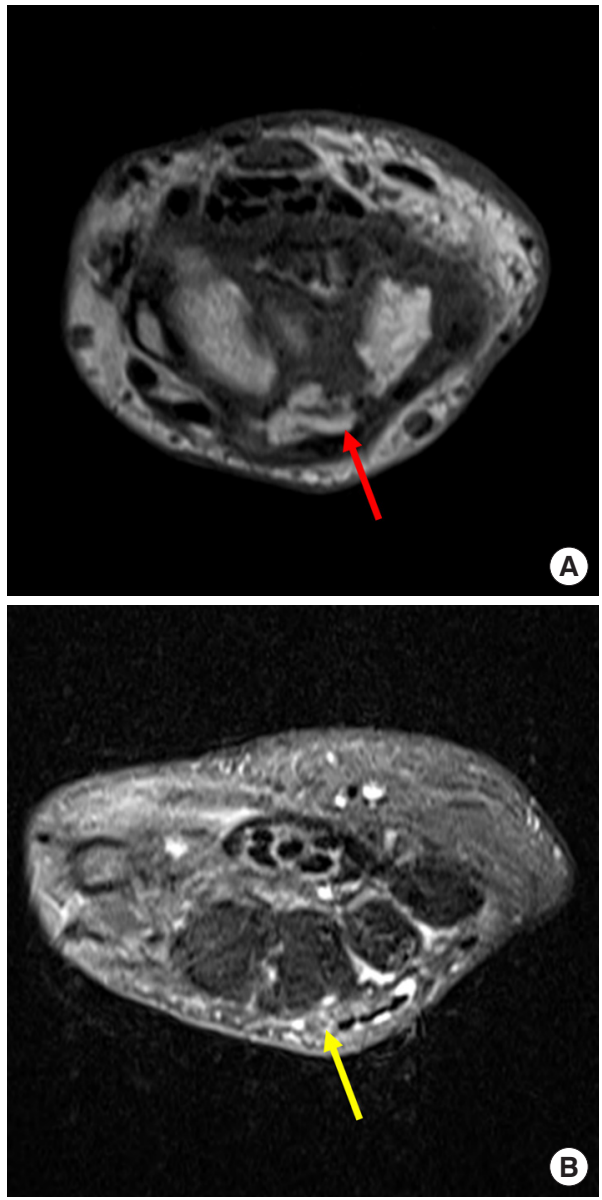

Fig. 2. Preoperative magnetic resonance images. (A) Degenerative changes of the intercarpal joints and dorsal displacement of the fragmented lunate bone in a T2 axial view (red arrow). (B) Complete tear of the extensor indicis proprius and second extensor digitorum communis tendons at the proximal level of the metacarpophalangeal joint in a $\mathrm{T} 1$ axial view (yellow arrow).
We planned transverse carpal ligament release for the carpal tunnel syndrome, and tendon transfer for the index finger of the extensor tendons with the fourth flexor digitorum superficialis (FDS) tendon. Intraoperatively, it was noted that all the extensor tendons in the index finger showed total rupture at the palmar level, and the proximal ends of the tendons could not be found. The surrounding tissues were scar-filled, caused by attritional inflammation. The distal stumps of the extensor tendons of the index finger were located $3 \mathrm{~cm}$ proximal to the corresponding metacarpophalangeal joints, and we harvested the fourth FDS tendon. The fourth FDS tendon was transferred to the dorsal side through a minimal incision and sutured with the EIP and second EDC tendons using the Pulvertaft weave method to perform tendon transfer (Fig. 3). The dislocated lunate bone was then removed. Two months after surgery at an outpatient follow-up visit, the patient was able to successfully lift her index finger through regular rehabilitation (Fig. 4).
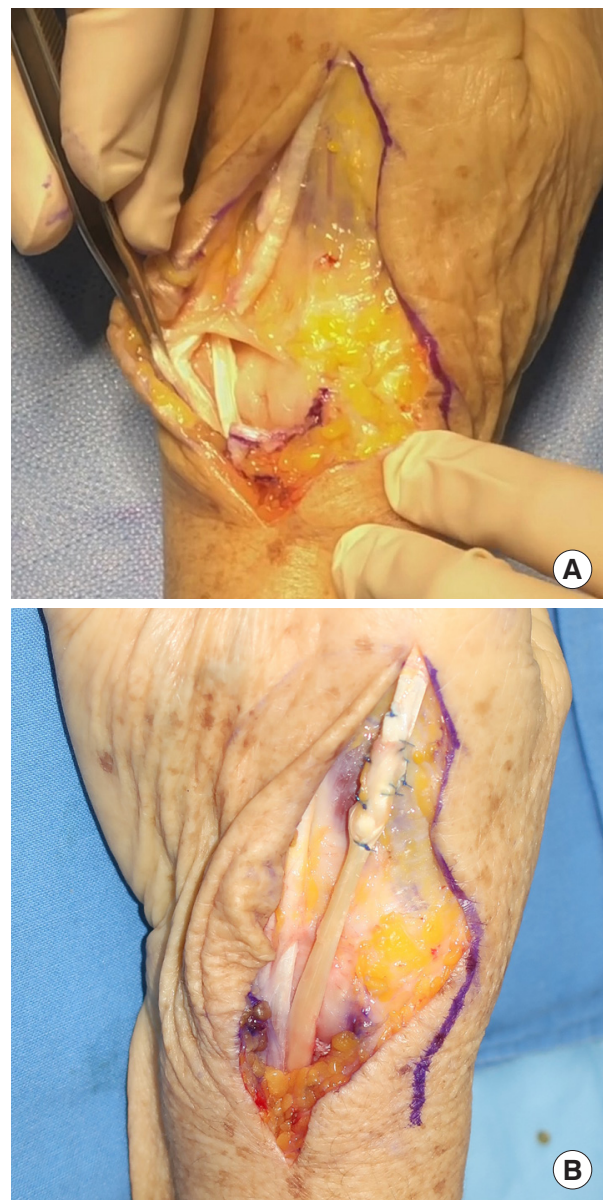

Fig. 3. Perioperative photograph. (A) Exposure of the fourth dorsal compartment revealed inflamed synovium around the ruptured second extensor digitorum communis tendon and the extensor indicis proprius tendons. (B) Tendon transfer through the fourth flexor digitorum superficialis tendon using the Pulvertaft weave method. 

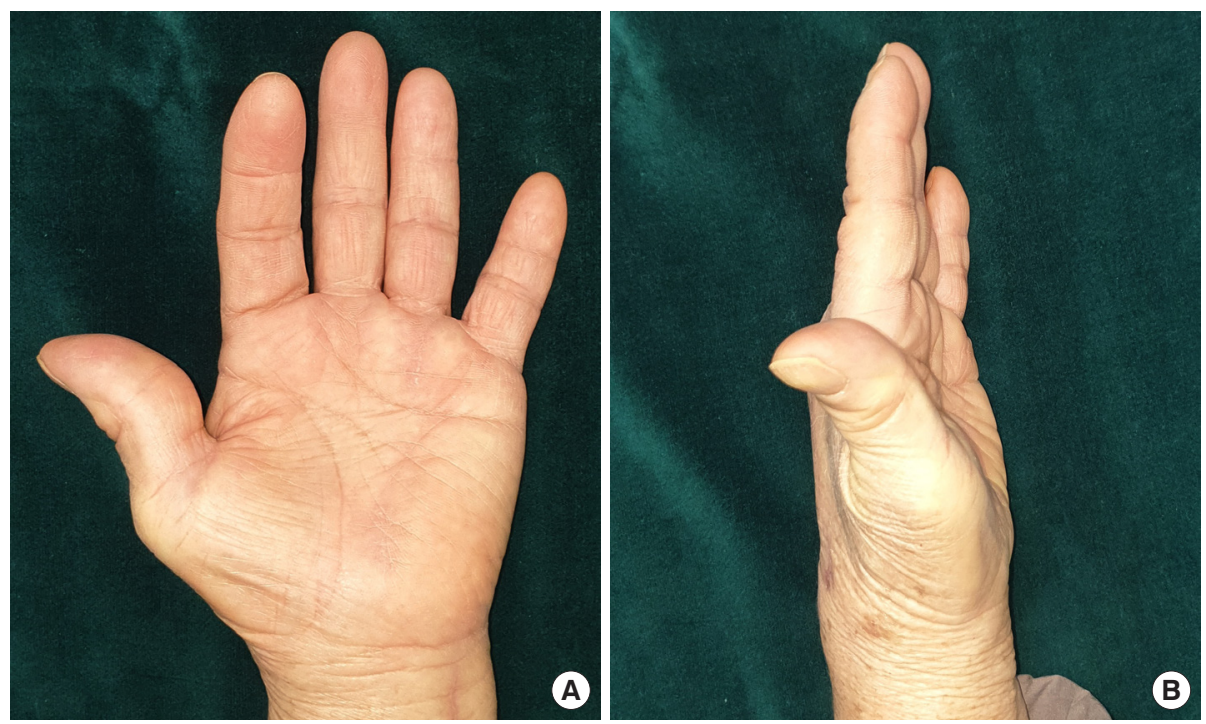

Fig. 4. The patient's left hand 8 weeks after surgery, without any complications. (A) Frontal and (B) lateral views.

\section{DISCUSSION}

Total rupture of the extensor tendon due to advanced Kienböck disease is very rare. The patient in our case, however, showed chronic Kienböck disease with dislocation of lunate bony fragments. In such cases, attritional ruptures can easily occur. Twenty-four cases of tendon rupture due to Kienböck disease have been reported.

Flexor tendon rupture was first reported by James in 1949, and extensor tendon rupture by Miki in 1986 [4].

Murase et al. [5] reported the recovery of extensor function of the index finger with a tendon graft using the palmaris longus tendon. Ramkumar et al. [3] reported the first case of severe fourth and fifth extensor tendon attrition and recovery of the range of motion of the injured fingers through tendon repair. Pacha-Vicente et al. [6] reported that multiple ruptures may occur in injuries of the extensor tendon of the index finger, and the extensor tendon of the little finger can be ruptured as a result of continuous friction with displaced lunate fragments.

Park et al. [7] reported ruptures of the third, fourth, and fifth extensor tendons caused by the posterior dislocation of lunate fragments due to advanced Kienböck disease in a 64-year-old male patient. To treat Kienböck disease, they removed the necrotic bone fragments of the lunate bone and performed scaphoid bone fusion. The EIP was transferred to the stump of the middle finger extensor tendon, and a palmaris longus tendon graft was sutured between the proximal and distal stumps of the ruptured ring finger extensor tendon.

Kang et al. [8] reported a case of rupture of the third and fourth extensor tendons that occurred due to displacement of the dorsal fragment of the lunate bone in a 73-year-old female patient with advanced Kienböck disease. Joint capsule rupture also occurred. The lunate fragment was removed, and a tendon graft was performed.

Hernandez-Cortes et al. reported that the association between Kienböck disease and tendon rupture may result in delayed treatment and poor prognosis due to improper preoperative imaging and physical examination [7,9]. Tomori et al. [2] reported a ruptured EDC tendon of the ring finger due to Kienböck disease in a patient who delayed treatment for 6 months because she lacked symptoms in her everyday functions.

Kim et al. [10] reported extensor tendon lesions that were frequently observed in advanced Kienböck disease and stated that these lesions differ from those caused by other conditions, such as rheumatoid arthritis, degenerative arthritis of the distal radial ulnar joint, or distal radius fractures.

The lunate bone, one of the carpal bones of the hand, plays a role in maintaining the overall hand structure. It is a proximal carpal bone and is partially involved in wrist movement. The lunate bone is anatomically located just above the fourth extensor compartment. Thus, lunate fracture and displacement lead to bony irregularities and attritional damage to the adjacent tendons. Continuous frictional damage eventually leads to various complications, such as ligament rupture and joint inflammation.

The location of a collapsed lunate fragment is the most important factor for tendon rupture. Tendon rupture associated with a collapsed lunate fragment occurs mainly in the fourth extensor compartment, which traverses the area of the lunate bone. It has been observed in several cases that damage occurs not only in the extensor ligament adjacent to the lunate bone, but also in 
other flexor tendons. For this reason, the second, third and fourth extensor tendons are usually damaged, but if lunate displacement is severe with multiple dislocations, the little finger extensor tendon can be damaged as well.

Our case involved a farmer who used both hands every day. The delayed diagnosis of Kienböck disease may have led to continuous damage of her tendons. Accumulated frictional damage may abruptly rupture a tendon. In our case, it is likely that this case of tendon rupture occurred due to the strong force required to pull radishes. When taking a patient's history, physicians should be sure to ask about lifestyle-related factors, such as age, gender, wrist pain, and occupational history. The differential diagnosis with other underlying diseases is important, especially rheumatic diseases. In the physical examination, both active and passive motion should be examined. Physicians should also check in the examination whether patients feel pain when moving their wrist and hand, and whether they can move their fingers well. Radiological tools can be used as initial examinations of bony lesions, and understanding the degree of necrosis based on Lichtman's radiographic classification is helpful for treatment planning. Other imaging studies, such as computed tomography or MRI to confirm tendon injuries, lunate displacement, and inflammatory signs are also useful when planning appropriate treatment.

\section{NOTES}

\section{Conflict of interest}

No potential conflict of interest relevant to this article was reported.

\section{Ethical approval}

The study was approved for exemption by the Institutional Review Board of Uijeongbu St. Mary's Hospital (IRB exemption No. 0210709-001).

\section{Patient consent}

The patient provided written informed consent for the publication and the use of her images.

\section{Author contribution}

Conceptualization: SN Jung. Data curation: JY Choi. Formal analysis: WJ Cha. Methodology: ER Jung. Project administra- tion: SN Jung. Visualization: WJ Cha, BF Seo. Writing - original draft: JY Choi. Writing - review \& editing: SN Jung. All authors read and approved the final manuscript.

\section{ORCID}

Jong Yun Choi https://orcid.org/0000-0002-1164-4499

Won Jin Cha https://orcid.org/0000-0003-0761-4251

Ee Room Jung https://orcid.org/0000-0001-6510-8045

Bommie F. Seo https://orcid.org/0000-0002-6907-5962

Sung-No Jung https://orcid.org/0000-0002-0419-4717

\section{REFERENCES}

1. Netscher DT, Badal JJ. Closed flexor tendon ruptures. J Hand Surg Am 2014;39:2315-23.

2. Tomori Y, Nanno M, Takai S. Closed rupture of extensor tendon resulting from untreated Kienböck disease: a case report and a review of the literature. Medicine (Baltimore) 2019;98:e16900.

3. Ramkumar S, Josty IC, Sykes PJ. Severe extensor tendon attrition and multiple tendon ruptures resulting from Kienböck's disease. Ann Plast Surg 2000;45:647-50.

4. Miki T, Yamamuro T, Kotoura Y, et al. Rupture of the extensor tendons of the fingers: report of three unusual cases. J Bone Joint Surg Am 1986;68:610-4.

5. Murase T, Ando Y, Hiroshima K. Extensor tendon rupture due to Kienböck's disease. J Hand Surg Br 1997;22:597-8.

6. Pacha-Vicente D, Sevilla-Tirado J, Lopez-Martinez R, et al. Extensor digiti minimi damage due to longstanding Kienböck's disease. J Hand Surg Eur Vol 2007;32:231.

7. Park JW, Kim SK, Park JH, et al. Multiple extensor tendon ruptures with advanced Kienböck's disease. J Hand Surg Am 2007;32:233-5.

8. Kang CH, Cho CH, Kim KW. Rupture of the extensor tendon secondary to advanced Kienböck's disease. J Korean Orthop Assoc 2012;47:59-63.

9. Hernandez-Cortes P, Pajares-Lopez M, Gomez-Sanchez R, et al. Rupture of extensor tendon secondary to previously undiagnosed Kienböck disease. J Plast Surg Hand Surg 2012;46:291-3.

10. Kim TG, Heo YM, Min YK. Extensor tendon rupture due to advanced Kienböck's disease: two case reports and a review of literature. J Hand Surg Asian Pac Vol 2020;25:123-8. 